\title{
Modeling oscillations and waves of cAMP in Dictyostelium discoideum cells
}

\author{
José Halloy*, Jacques Lauzeral, Albert Goldbeter \\ Faculté des Sciences, Université Libre de Bruxelles, Campus Plaine, C.P. 231, B-1050 Brussels, Belgium
}

Revision received 22 January 1998; accepted 13 February 1998

\begin{abstract}
We examine the theoretical aspects of temporal and spatiotemporal organization in the cAMP signaling system of Dictyostelium discoideum amoebae which aggregate in a wavelike manner after starvation, in response to pulses of cAMP emitted with a periodicity of several minutes by cells behaving as aggregation centers. We first extend the model based on receptor desensitization, previously proposed by Martiel and Goldbeter, by incorporating the role of $\mathrm{G}$ proteins in signal transduction. The extended model accounts for observations on the response of the signaling system to successive step increases in extracellular cAMP. In the presence of the positive feedback loop in cAMP synthesis, this model generates sustained oscillations in cAMP and in the fraction of active cAMP receptor, similar to those obtained in the simpler model where the role of the $\mathrm{G}$ proteins is not taken into account explicitly. We use the latter model to address the formation of concentric and spiral waves of cAMP in the course of $D$. discoideum aggregation. Previous analyses of the model showed that a progressive increase in the activity of adenylate cyclase and phosphodiesterase can account for the transitions no relayrelay-oscillations-relay observed in the experiments. We show that the degree of cellular synchronization on such a developmental path in parameter space markedly affects the nature of the spatial patterns generated by the model. These patterns range from concentric waves to a small number of large spirals, and finally to a large number of smaller spirals, as the degree of developmental desynchronization between cells increases. () 1998 Elsevier Science Ireland B.V. All rights reserved
\end{abstract}

Keywords: Modeling; Oscillations; Waves; cAMP; Dictyostelium discoideum

\section{Introduction}

Few cellular types have contributed as much as Dictyostelium discoideum amoebae to the experimental and theoretical study of spatiotemporal organization at the supracellular level. After starvation, these

\footnotetext{
* Corresponding author. Tel.: +32 2 6505770; fax: +32 2 6505767; e-mail: jhalloy@ ulb.ac.be
}

cells stop growing and dividing, and embark on a developmental program that includes their aggregation by chemotaxis toward signals of cAMP emitted by cells behaving as aggregation centers, and the subsequent transformation of this multicellular aggregate, which can contain up to $10^{5}$ cells, into a fruiting body surmounted by a mass of spores. The life cycle resumes after the spores have been dispersed in the environment [1-3].

The aggregation of $D$. discoideum cells possesses a 
periodic nature: on a solid support, the amoebae collect around centers in waves, as a result of the pulsatile release of cAMP signals by the centers with a periodicity of 5-10 min [4-7]. Experiments in cell suspensions have confirmed the capability of aggregation centers to release cAMP signals in a periodic manner [8]. These experiments further showed that in addition to such pacemaker behavior, the amoebae are also capable of excitability, i.e. of relaying suprathreshold cAMP pulses $[9,10]$.

The mechanism for the periodic generation of cAMP signals involves a positive feedback loop in the synthesis of cAMP: indeed, extracellular cAMP binds to a cell surface receptor and thereby activates adenylate cyclase which synthesizes cAMP from ATP [11]. Intracellular cAMP is then secreted into the extracellular medium where it binds to its receptor and further promotes its own synthesis. At least two processes limit the effect of such autocatalysis. First, cAMP is degraded by phosphodiesterase; second, binding of cAMP induces desensitization of the cAMP receptor. The latter phenomenon is accompanied by reversible phosphorylation of the receptor $[12,13]$. A model based on receptor desensitization has been proposed for cAMP relay and oscillations in suspensions of $D$. discoideum cells $[11,14]$. This three-variable model has also been used in the theoretical study of cAMP waves in the course of aggregation [15-17].

The model based on receptor desensitization does not include the role of $G$ proteins which act as signal transducers between cAMP binding to the receptor and the subsequent activation or inactivation of adenylate cyclase $[18,19]$. Thus, experiments indicate in D. discoideum $[20,21]$ that a $\mathrm{G}_{\mathrm{s}}$ protein mediates the activation of the cyclase, whereas a $\mathrm{G}_{\mathrm{i}}$ protein might perform an opposite function, by inhibiting the enzyme. The positive control of adenylate cyclase via $G$ proteins remains at the moment clearer than the inhibitory part of the regulatory network, which remains largely hypothetical [22-24].

The purpose of this work is two-fold. First, we wish to incorporate the role of $\mathrm{G}$ proteins into the model for cAMP signaling based on receptor desensitization. Our approach $[11,25]$ is closely related to that proposed by Tang and Othmer [26]. We show that the extended model accounts better than the former version for the experiments of Devreotes and Steck who investigated the response of the signaling system to successive increments in extracellular cAMP, in conditions where the positive feedback loop is suppressed [27]. Although the conditions are somewhat unphysiological, these experiments carried out in the absence of positive feedback are of great interest since they show how the system operates under a different set of constraints. The study of the extended model further indicates that it is capable of sustained oscillatory behavior $[11,25]$. These results, however, do not provide significantly new insights with respect to the predictions of the simpler model as to relay and oscillations which represent the major physiological responses. Therefore, the original, threevariable model still encompasses the key aspects of cAMP relay and oscillations based on positive feedback and receptor desensitization.

The second goal of this study is to investigate, by means of the three-variable model based on receptor desensitization, the origin of concentric and spiral waves of cAMP in the course of $D$. discoideum aggregation [3-7]. Concentric waves arise when a pacemaker releases periodic cAMP signals in a field of excitable cells. If obtaining concentric waves in theoretical models in such a way is rather straightforward, spiral waves, in contrast, are often generated in a largely artificial manner $[15,17,28]$. Thus, taking initial conditions which correspond to the breaking of a planar or circular wave readily produces spirals. It is much more difficult to trigger the spontaneous formation of spiral waves. In several studies, random pulses of cAMP have been used to generate such waves, in addition to inhomogeneities associated with or amplified by cell movement $[29,30]$ or the inhomogeneous distribution of the extracellular inhibitor acting on phosphodiesterase [31].

We have recently proposed an alternative, developmentally-based scenario for the onset of spiral waves of cAMP [17]. The analysis of the model shows that the continuous increase in the activity of adenylate cyclase and phosphodiesterase after starvation [32] brings the cells from a domain of no relay to a domain of relay, i.e. excitability, and then to the domain of sustained, autonomous oscillations, before the cells return to the excitable state. These changes in the activity of the two enzymes define a developmental path that cells follow in parameter space after starvation $[33,34]$. We showed that the desynchronization of 
cells on the developmental path can trigger the appearance of defects which lead to the formation of cAMP spirals [17]. Here we extend these results by studying how the magnitude of cellular desynchronization affects the type of wave that spontaneously forms. To obtain better insight into the origin of spirals, we present a detailed sequence of spatiotemporal patterns observed on the way from concentric to spiral waves of cAMP.

\section{Model for cAMP signaling based on receptor desensitization}

The original version of the model for cAMP signaling based on receptor desensitization proposed by Martiel and Goldbeter is shown in Fig. 1 [14]. The model incorporates binding of extracellular cAMP to the active (R) and desensitized (D) forms of the receptor; in the absence of the ligand the amount of receptor in the active state is much larger than in the desensitized state, while the reverse situation obtains after a sufficiently long incubation of the receptor with cAMP. Also included in the model is the activation of adenylate cyclase by collision with the complex formed by cAMP with the active form of the receptor, and the hydrolysis of cAMP by the intracellular and extracellular forms of phosphodiesterase. The interconvertible $\mathrm{R}$ and $\mathrm{D}$ states can be viewed as different conformations or as the dephosphorylated and phosphorylated forms of the receptor [12-14].

The time evolution of the model schematized in Fig. 1 is governed by a set of non-linear kinetic equations which can be reduced to three or even two variables. These equations are listed in Ref. [14] and also in Section 4 below. The numerical analysis of these equations shows that the model can account in a qua-

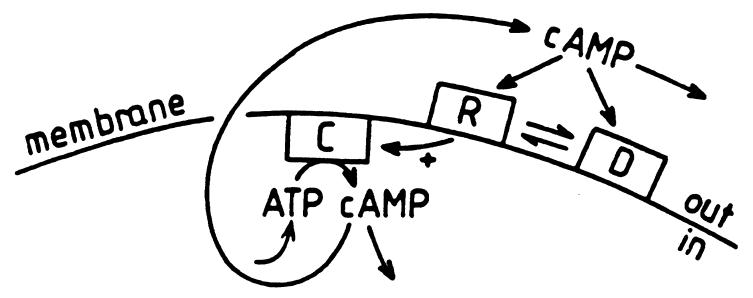

Fig. 1. Basic model for cAMP signaling in Dictyostelium cells, involving self-amplification in cAMP synthesis as well as receptor desensitization (see Refs. [11,14]). litative and quantitative manner for most experiments on relay and oscillations of cAMP in cell suspensions. Applying the model to the study of spatiotemporal patterns requires the inclusion of a diffusion term for extracellular cAMP, as considered elsewhere [15-17] and in Section 4 below.

The model, however, does not account well for the response of the signaling system to successive increments of cAMP, in conditions where the positive feedback loop is suppressed $[11,25,26]$. Although these conditions are somewhat unnatural, they yield interesting insights into the dynamics of the signaling system and provide constraints on theoretical models proposed for cAMP synthesis in D. discoideum. To better account for this type of experiment, it is necessary to go into further detail regarding the mechanism of signal transduction. This requires incorporating the role of $\mathrm{G}$ proteins in the control of adenylate cyclase, following binding of extracellular cAMP to its membrane receptor.

\section{Incorporation of $G$ proteins into the model for cAMP signaling}

Experiments suggest that $G$ proteins exert a dual control on adenylate cyclase. While in the model schematized in Fig. 1 the enzyme is activated directly upon collision with the complex formed by cAMP with the active (dephosphorylated) form of the receptor, experiments have shown $[20,21]$ that this activation is mediated by a protein, member of the $G_{s}$ family. On the other hand, desensitization of the cAMP receptor, accompanied by phosphorylation in the model of Fig. 1, is the major process responsible for termination of the cAMP response: the desensitized receptor was assumed to be unable to activate adenylate cyclase. The experiments suggest however, that the desensitized receptor might activate a $\mathrm{G}_{\mathrm{i}}$ protein which would, in turn, inhibit adenylate cyclase $[20,21]$. The model incorporating such roles for the $G_{s}$ and $G_{i}$ proteins is shown in Fig. 2. Upon collision with the cAMP-R complex, the $\mathrm{G}_{\mathrm{s}}$ protein releases an active moiety which then activates, by collision coupling, a molecule of adenylate cyclase. Conversely, upon collision with the cAMP-D complex, the $\mathrm{G}_{\mathrm{i}}$ protein releases an active moiety which binds to, and thereby inhibits, adenylate cyclase. 
With respect to the previous version of the model based on receptor desensitization, the extended version of Fig. 2 contains two additional variables, namely the fractions $g_{s}$ and $g_{i}$ of active subunits of the $G_{s}$ and $G_{i}$ proteins. The time evolution of the cAMP signaling system is now governed by the system of five non-linear kinetic Eq. (1):

$$
\begin{aligned}
& \frac{\mathrm{d} \rho_{\mathrm{T}}}{\mathrm{d} t}=-\rho_{\mathrm{T}} f_{1}(\gamma)+\left(1-\rho_{\mathrm{T}}\right) f_{2}(\gamma) \\
& \frac{\mathrm{d} g_{\mathrm{s}}}{\mathrm{d} t}=\varphi_{1} x\left(\rho_{\mathrm{T}}, \gamma\right)\left(1-g_{\mathrm{s}}\right)-k_{3}^{\prime} g_{\mathrm{s}} \\
& \frac{\mathrm{d} g_{\mathrm{i}}}{\mathrm{d} t}=\varphi_{2} y\left(\rho_{\mathrm{T}}, \gamma\right)\left(1-g_{\mathrm{i}}\right)-k_{4}^{\prime} g_{\mathrm{i}} \\
& \frac{\mathrm{d} \beta}{\mathrm{d} t}=\sigma q \Phi\left(g_{\mathrm{s}}, g_{\mathrm{i}}\right)-\left(k_{\mathrm{i}}+k_{\mathrm{t}}\right) \beta \\
& \frac{\mathrm{d} \gamma}{\mathrm{d} t}=\frac{k_{\mathrm{t}}}{h} \beta-k_{\mathrm{e}} \gamma
\end{aligned}
$$

with

$$
\begin{aligned}
& f_{1}(\gamma)=\frac{k_{1}+k_{2} \gamma}{1+\gamma}, f_{2}(\gamma)=\frac{k_{-1}+k_{-2} c \gamma}{1+c \gamma} \\
& x\left(\rho_{\mathrm{T}}, \gamma\right)=\frac{\rho_{\mathrm{T}} \gamma}{1+\gamma}, y\left(\rho_{\mathrm{T}}, \gamma\right)=\frac{\left(1-\rho_{\mathrm{T}}\right) c \gamma}{1+c \gamma}, \\
& \Phi\left(g_{\mathrm{s}}, g_{\mathrm{i}}\right)=\frac{\alpha\left(\frac{g_{\mathrm{s}}^{2}}{\epsilon_{\mathrm{s}}}+\lambda \theta\right)}{1+\theta \lambda+(1+\alpha) \frac{g_{\mathrm{s}}^{2}}{\epsilon_{\mathrm{s}}}+\frac{g_{\mathrm{i}}^{2}}{\epsilon_{\mathrm{i}}}}
\end{aligned}
$$

In these expressions, $\rho_{\mathrm{T}}$ denotes the total fraction of active cAMP receptor, while $g_{\mathrm{s}}$ and $g_{\mathrm{i}}$ are the fractions of active $\mathrm{G}_{\mathrm{s}}$ and $\mathrm{G}_{\mathrm{i}}$ proteins; $\beta$ and $\gamma$ denote the normalized concentrations of intracellular and extracellular cAMP; $\epsilon_{\mathrm{s}}$ and $\epsilon_{\mathrm{i}}$ are dissociation constants for the coupling of $\mathrm{G}_{\mathrm{s}}$ and $\mathrm{G}_{\mathrm{i}}$ to adenylate cyclase, divided by the total amounts of $\mathrm{G}_{\mathrm{s}}$ and $\mathrm{G}_{\mathrm{i}}$, respectively; $\varphi_{1}$ and $\varphi_{2}$ are bimolecular rate constants, multiplied by the total amount of receptor, for the activation of $\mathrm{G}_{\mathrm{s}}$ and $\mathrm{G}_{\mathrm{i}}$ by collision with the cAMP-receptor complex in the $\mathrm{R}$ or D state (see Ref. [14] for further details on the definition of other parameters).

In the model, we assume that the active (R) and desensitized (D) states correspond to unphosphory-

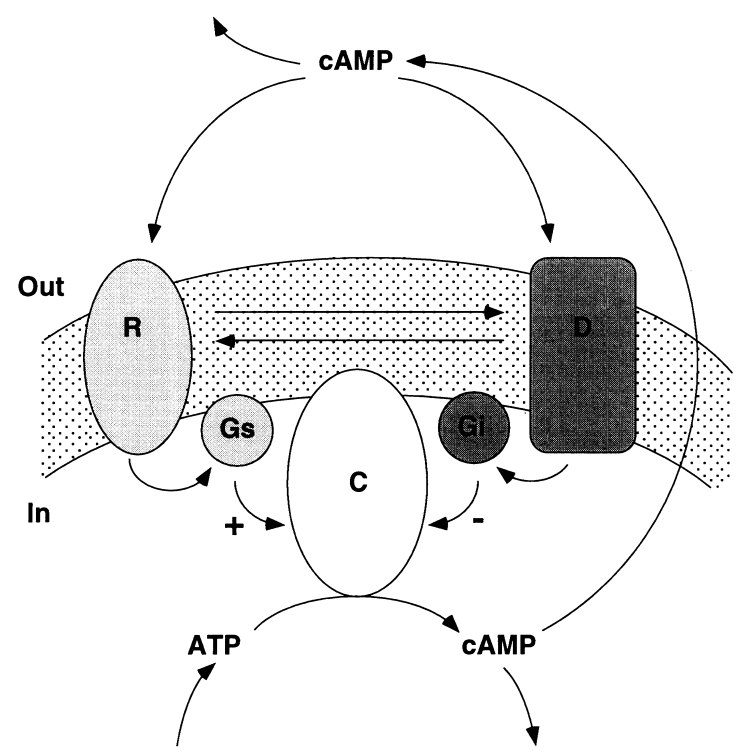

Fig. 2. Model for cAMP signaling incorporating the effect of $G$ proteins. As in the basic model shown in Fig. 1, the receptor is considered to exist in two interconvertible states, active (R) and desensitized (D), which could represent the dephosphorylated and phosphorylated forms, respectively. Upon binding of cAMP, the R forms activates adenylate cyclase $(C)$ via the action of a $G_{s}$ protein, while the desensitized form inhibits adenylate cyclase via the action of a protein $\mathrm{G}_{\mathrm{i}}$. The rest of the model is similar to that of Fig. 1 with respect to the synthesis and transport of intracellular cAMP into the extracellular medium, and with respect to hydrolysis of intracellular and extracellular cAMP by phosphodiesterase.

lated and phosphorylated states of the receptor, respectively. The precise role of phosphorylation in receptor desensitization remains, however, unclear. Phosphorylation appears to stabilize the desensitized state, but experimental observations point to the existence of a back-up mechanism for adaptation, that could bypass receptor phosphorylation [24].

The expressions in Eq. (2) have been obtained by assuming that the activation and inhibition of adenylate cyclase result from the collision of the enzyme with two molecules of the active forms of $G_{s}$ and $G_{i}$, respectively [25]. Such non-linearities (which could be replaced by cooperative binding of cAMP to its receptor) are necessary to generate sustained oscillations. Also considered in the model is the inactivation of the $\mathrm{G}_{\mathrm{s}}$ and $\mathrm{G}_{\mathrm{i}}$ active moieties due to the intrinsic GTPase activity of these proteins $[18,19]$; the linear inactivation of $\mathrm{G}_{\mathrm{s}}$ and $\mathrm{G}_{\mathrm{i}}$ in Eq. (1) is characterized by the rate constants $k_{3}^{\prime}$ and $k_{4}^{\prime}$, respectively. 


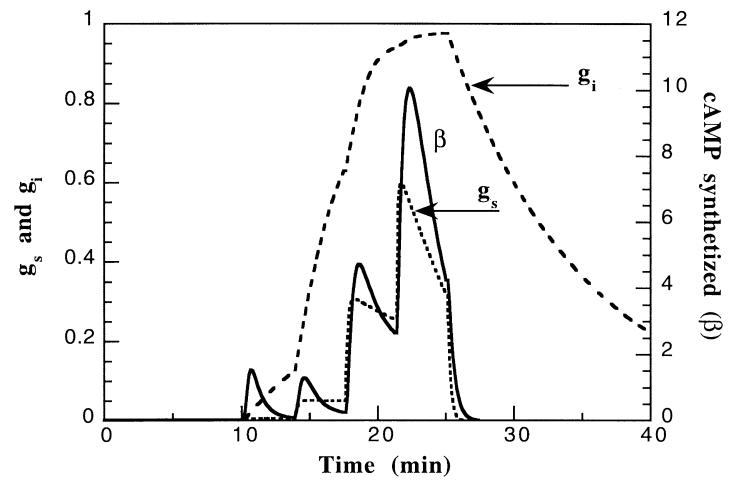

Fig. 3. Response to successive increments in extracellular cAMP, predicted by the model incorporating the control of adenylate cyclase by the $\mathrm{G}_{\mathrm{s}}$ and $\mathrm{G}_{\mathrm{i}}$ proteins. The concentration of extracellular cAMP is successively raised from 0 to $10^{-9} \mathrm{M}, 10^{-8} \mathrm{M}, 10^{-7} \mathrm{M}$ and $10^{-6} \mathrm{M}$ in 4 steps of $225 \mathrm{~s}$ duration each. Shown are the changes in the amount of intracellular $\operatorname{cAMP}(\beta)$ secreted and in the fractions of active $\mathrm{G}_{\mathrm{s}}$ and $\mathrm{G}_{\mathrm{i}}$ proteins elicited by these sequential stimuli. The data is obtained by numerical integration of Eq. (1) where $\gamma$ becomes a controlled parameter rather than a variable. Parameters values are $\varphi_{1}=25 \mathrm{~min}^{-1}, \varphi_{2}=5 \mathrm{~min}^{-1}, \epsilon_{\mathrm{s}}=10^{-3}$, $\epsilon_{\mathrm{i}}=10^{-4}, k_{3}^{\prime}=5 \mathrm{~min}^{-1}, k_{4}^{\prime}=0.1 \mathrm{~min}^{-1}$; other parameter values are given in Table II of Ref. [14].

\subsection{Response to successive increments of extracellular cAMP}

The basic version of the model (Fig. 1) fails to account in a satisfactory manner $[11,25,26]$ for a particular set of experiments carried out in conditions where the level of extracellular cAMP is controlled and raised in successive increments lasting $225 \mathrm{~s}$ [27]. The model incorporating the two $G$ proteins fares better in this respect, as indicated by the data of Fig. 3 showing the cAMP synthesis triggered by four successive step increases in extracellular cAMP, first from zero to $10^{-9} \mathrm{M}$, and then from $10^{-9} \mathrm{M}$ to $10^{-6}$ $M$ in three successive, ten-fold increments. Also shown in Fig. 3 are the associated changes in the fractions of active $G_{s}$ and $G_{i}$ proteins. Whereas the former version of the model failed to respond to the first increment and produced glued responses to the three following increases in cAMP, the version incorporating the $G$ proteins yields four separated responses.

The fact that the second peak in intracellular cAMP can be smaller than the first and the third and fourth ones, as in the case illustrated in Fig. 3, fits with the experimental observations (see Fig. 8 in Ref. [27]). This phenomenon, which is not obtained for all parameter values, results from a delicate balance between the inactivation kinetics of $\mathrm{G}_{\mathrm{s}}$ and $\mathrm{G}_{\mathrm{i}}$. The model indicates that to obtain better agreement with the experimental observations and, in particular, a good separation between the four successive responses, the inactivation of $\mathrm{G}_{\mathrm{s}}$ must be faster, by at least a factor of 5 , than that of $\mathrm{G}_{\mathrm{i}}$. This requirement appears to hold with experimental observations [21].

\subsection{Oscillations and relay of cAMP signals}

The experiments addressed in Section 3.1 are related to conditions in which the positive feedback in cAMP synthesis is suppressed. When this regulatory loop is restored as in normal, physiological conditions, sustained oscillations are observed in the model (Fig. 4). For the same set of parameter values as in Fig. 3, the model then generates limit cycle oscillations with a period of about 12 min which match those observed in the experiments.

As in the original version of the model based on receptor desensitization, cAMP oscillations are accompanied by the periodic alternance of the receptor between the active and desensitized states [13]. Moreover, the rise in intracellular cAMP in Fig. 4 is concomitant with an increase in the fractions of active

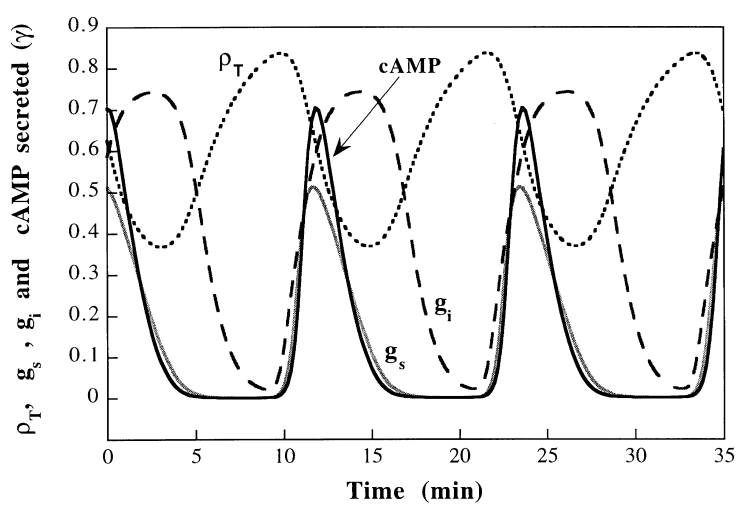

Fig. 4. Sustained oscillations predicted by the model incorporating the control of adenylate cyclase by the $\mathrm{G}_{\mathrm{s}}$ and $\mathrm{G}_{\mathrm{i}}$ proteins. Shown is the variation in the normalized concentration of extracellular $\operatorname{cAMP}(\gamma)$ and in the fractions of active receptor $\left(\rho_{\mathrm{T}}\right)$ and of active $\mathrm{G}_{\mathrm{s}}$ and $\mathrm{G}_{\mathrm{i}}$ proteins. The curves are generated by numerical integration of Eq. (1), where $\gamma$ is a variable, for the parameter values of Fig. 3, with $k_{4}^{\prime}=1$ (instead of 0.1$) \mathrm{min}^{-1}$. 
$\mathrm{G}_{\mathrm{s}}$ and $\mathrm{G}_{\mathrm{i}}$; the decline in the fraction $g_{\mathrm{s}}$ occurs at the same time as the decrease in cAMP, whereas the drop in $g_{\mathrm{i}}$ appears to be delayed in the case considered, and contributes to enlarging the interspike interval. However, the oscillations in cAMP and in the fraction of active receptor appear to be similar to those generated by the simpler, three-variable model $[11,14]$ in which the detailed role of the $\mathrm{G}$ proteins is not taken into account explicitly. Much as in the latter model, for parameter values corresponding to a stable, excitable steady state, the extended model can relay suprathreshold pulses of extracellular cAMP (data not shown). Oscillations and relay can still occur in the extended model in the absence of $\mathrm{G}_{\mathrm{i}}$, when only the role of $\mathrm{G}_{\mathrm{s}}$ is taken into account.

\section{Origin of concentric and spiral waves of cAMP}

The excitable and oscillatory dynamics of the cAMP signaling system lies at the heart of the propagation of concentric or spiral waves of cAMP in the course of $D$. discoideum aggregation. To investigate the origin of these spatiotemporal patterns, we return to the three-variable model for cAMP relay and oscillations and include the diffusion of extracellular cAMP to address the formation of concentric and spiral waves of cAMP. The spatiotemporal evolution of the three-variable system is now governed by the system of Eq. (3):

$$
\begin{aligned}
& \frac{\mathrm{d} \rho_{\mathrm{T}}}{\mathrm{d} t}=-f_{1}(\gamma) \rho_{\mathrm{T}}+f_{2}(\gamma)\left(1-\rho_{\mathrm{T}}\right) \\
& \frac{\mathrm{d} \beta}{\mathrm{d} t}=q \sigma \phi\left(\rho_{\mathrm{T}}, \gamma \alpha\right)-\left(k_{\mathrm{i}}+k_{\mathrm{t}}\right) \beta \\
& \frac{\partial \gamma}{\partial t}=\left(k_{\mathrm{t}} \beta / h\right)-k_{e} \gamma+D_{\gamma} \nabla^{2} \gamma
\end{aligned}
$$

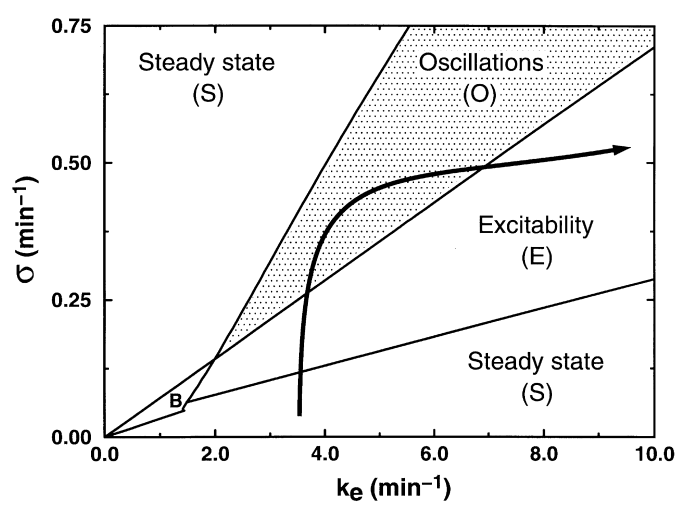

Fig. 5. Developmental path (arrow) followed by Dictyostelium cells after starvation. The arrow passes successively through the domains of stable steady states (S), excitability (E), autonomous, sustained oscillations $(\mathrm{O})$, to end, again, in a region of excitability. The latter is determined numerically as the capability of the system to propagate a cAMP wave in space without attenuation. The developmental path is represented here as a function of the changes in two of the main control parameters after starvation: adenylate cyclase $(\sigma)$ and phosphodiesterase $\left(k_{\mathrm{e}}\right)$. The diagram is established for the Martiel-Goldbeter model [14] governed by Eq. (3), for the parameters values listed in Fig. 1 of Ref. [17].

with

$f_{1}(\gamma)=\frac{k_{1}+k_{2} \gamma}{1+\gamma}, f_{2}(\gamma)=\frac{k_{1} L_{1}+k_{2} L_{2} c \gamma}{1+c \gamma}$

$\phi\left(\rho_{\mathrm{T}}, \gamma, \alpha\right)=\frac{\alpha\left(\lambda \theta+\epsilon Y^{2}\right)}{1+\alpha \theta+\epsilon Y^{2}(1+\alpha)}, Y=\frac{\rho_{\mathrm{T}} \gamma}{1+\gamma}$

In contrast to other studies [16,29,31], these equations do not include the slower chemotactic movement of the cells, since at this stage we wish to focus on the reaction-diffusion mechanism responsible for generating the different types of cAMP waves.

Fig. 6. Effect of cellular desynchronization on the type of cAMP wave generated by the model for cAMP signaling based on receptor desensitization. The data are obtained by numerical integration of Eq. (3), taking into account the temporal variation of the activities of adenylate cyclase and phosphodiesterase. The equations governing the changes in parameters $\sigma$ and $k_{\mathrm{e}}$ associated with the developmental path depicted in Fig. 5 are given in Fig. 4 of Ref. [17]. We assume that the cells begin their journey on the developmental path at starting times $t_{\mathrm{s}}$ randomly distributed in space, according to the probability distribution $P\left(t_{\mathrm{s}}\right)$ given by Eq. (5), in which the degree of desynchronization $\Delta$ measures the dispersion of $P\left(t_{\mathrm{s}}\right)$. Panels a, $\mathrm{b}$, and $\mathrm{c}$ are established for $\Delta=15,25$ or $100 \mathrm{~min}$, respectively. The upper rows show the spatial distribution of extracellular cAMP $(\gamma)$ at the times $0,150,240,270$ and $360 \mathrm{~min}$ after the beginning of the increase in $\sigma$ and $k_{\mathrm{e}}$. The bottom rows show the distribution of cells along the developmental path of Fig. 5 (each circle represents $5 \%$ of the total number of cells) at the corresponding times. The figures are generated by numerical integration of Eq. (4) on a spatial grid of $100 \times 100$ points representing an area of $1 \mathrm{~cm}^{2}$ (see [17] for further details). Parameter values are the same as in Fig. 5. 


\subsection{The developmental path}

Earlier theoretical studies showed that a progressive increase in the activity of adenylate cyclase and phosphodiesterase [32] can account for the transitions no relay-relay-oscillations-relay observed in the experiments in cell suspensions $[11,33,34]$. Thus, cells experiencing such variations in the levels of the two enzymes in the course of time follow a developmental path in the adenylate cyclase-phosphodiesterase parameter space [33], as illustrated in Fig. 5 for the model based on receptor desensitization. This

(a) $\Delta=15 \mathrm{~min}$

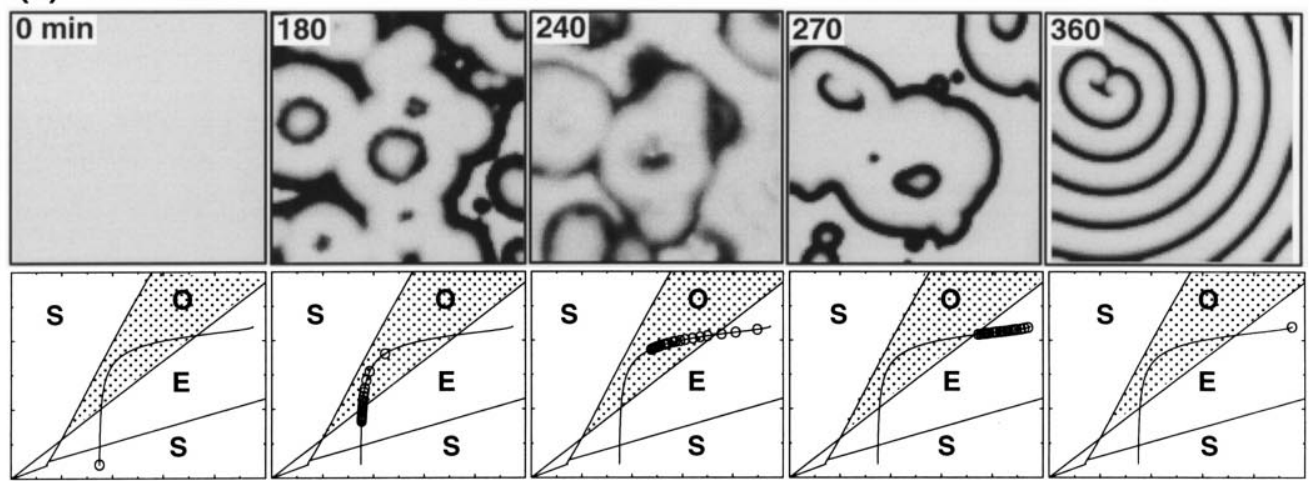

(b) $\Delta=25 \mathrm{~min}$

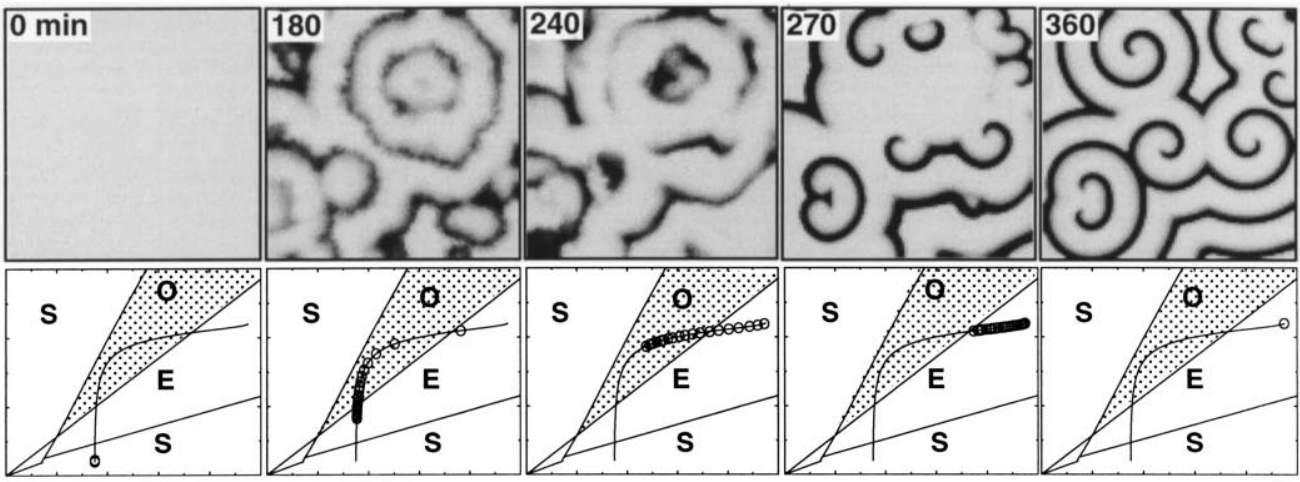

(c) $\Delta=100 \mathrm{~min}$

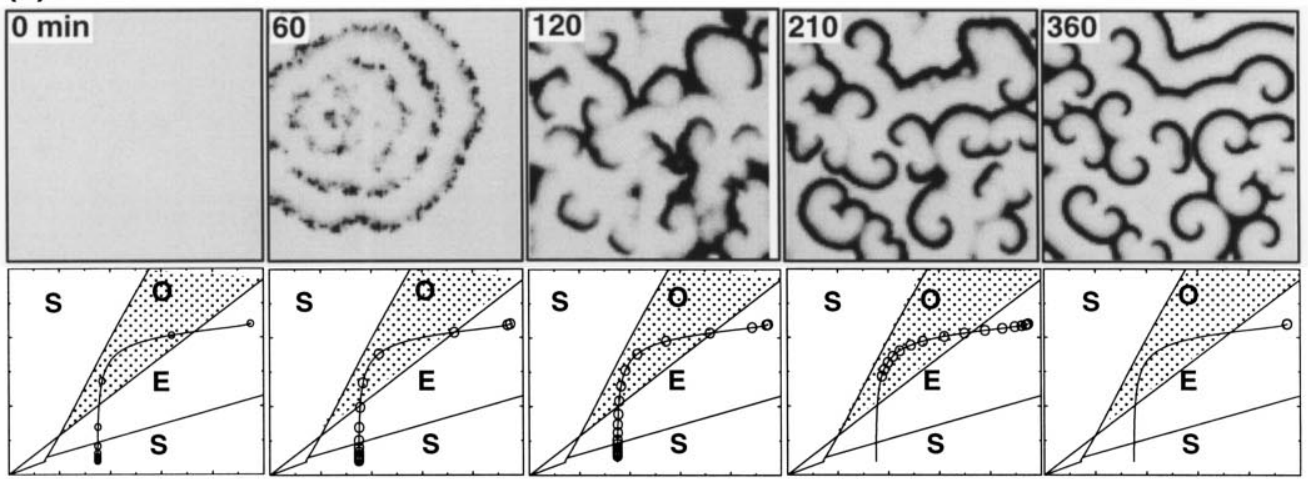


path, symbolized by the arrow in Fig. 5, brings the cells successively from a domain of stable, non-excitable steady states $(\mathrm{S})$ into the domain of relay, i.e. excitability (E), and then into the domain of autonomous, sustained oscillations $(\mathrm{O})$ before the system returns to an excitable state.

The time evolution of parameters $\sigma$ and $k_{\mathrm{e}}$ obeys equations which ensure that both enzyme activities increase in time in a sigmoidal manner, with phosphodiesterase lagging behind adenylate cyclase, as observed in the experiments. The analysis of Eq. (3) brings to light the possible role of the desynchronization of cells in the onset of spiral waves [17].

\subsection{Influence of cellular desynchronization on cAMP waves}

We have recently shown [17] that the degree of cellular desynchronization on such a developmental path in parameter space markedly affects the nature of the spatial patterns generated by the model. Cells do not start their developmental journey at the same position on the path. This amounts to considering a probability distribution for the starting time $t_{\mathrm{s}}$, corresponding to a particular pair of values of parameters $\sigma$ and $k_{\mathrm{e}}$, given by Eq. (5):

$P\left(t_{\mathrm{s}}\right)=(1 / \Delta) \exp \left(-t_{\mathrm{s}} / \Delta\right)$

The spatial distribution of starting times is taken randomly according to the probability distribution (Eq. (5)). Parameter $\Delta$ measures the degree of desynchronization of cells on the developmental path.

When the degree of desynchronization is relatively weak, e.g. $\Delta=15 \mathrm{~min}$, the cAMP patterns which progressively form tend to be concentric (Fig. 6a). At higher desynchronization, e.g. for $\Delta=25 \mathrm{~min}$ (as in the case considered also in Fig. 6 of Ref. [17]), a few large spirals are eventually observed (Fig. 6b). In contrast, at much higher degrees of desynchronization, e.g. for $\Delta=100 \mathrm{~min}$, this pattern transforms into a much larger number of smaller spirals (Fig. 6c).

To characterize in further detail the origin of spiral waves, it is useful to focus on the sequence of events leading to the formation of a typical spiral pattern. The data of Fig. 7 show the spatial distribution of extracellular cAMP at 10-min intervals, starting 150 min after movement on the developmental path has begun (before that time, the spatial inhomogeneities remain reduced). The corresponding evolution of the parameters can be inferred from the lower panel in Fig. $6 b$, established for the same value of $\Delta$, where each circle represents the position of $5 \%$ of the total amount of cells proceeding on the path. The sequence of frames shows that several concentric waves initially form (at about $160 \mathrm{~min}$ ) as the cells begin to enter the oscillatory domain, while other cells remain excitable. These waves are progressively broken by the defects which accumulate due to the spatial heterogeneity in the evolving parameter values. The breaks occur as cells progressively move out of the oscillatory domain, back into the excitable range (from 180 min to about $260 \mathrm{~min}$ ). Spirals eventually develop from the fragments of such broken waves.

\section{Discussion}

In the present paper we have addressed by means of theoretical models both the temporal and the spatiotemporal aspects of cAMP signaling in Dictyostelium cells. In the first part we focused on the temporal dynamics and incorporated explicitly into the model for cAMP signaling based on receptor desensitization the role of a $G_{s}$ and a $G_{i}$ protein which, respectively, activates and inhibits adenylate cyclase. The extended model, which contains five instead of three variables, accounts for the response of the signaling system to successive increments of extracellular cAMP when the latter stimulus is controlled in such a manner that the positive feedback loop in cAMP synthesis is suppressed. When this feedback is restored, the extended model accounts for sustained oscillations of cAMP, but the predictions of the model do not differ significantly from those of the basic, three-variable model in these physiological conditions.

The results generated by the model incorporating the effect of the $G_{s}$ and $G_{i}$ proteins are similar to those obtained by Tang and Othmer [26] in a closely related study which also includes the control of adenylate cyclase by $\mathrm{G}_{\mathrm{s}}$ and $\mathrm{G}_{\mathrm{i}}$, although the assumptions underlying the latter model differ from those considered here in at least two respects: there, rather than inhibiting directly adenylate cyclase, $G_{i}$ is assumed to act indirectly by preventing the activation of $G_{s}$ which follows its collision with the complex formed by cAMP bound to the active receptor form. Moreover, 
the authors consider two forms of the cAMP receptor, one which activates $G_{s}$ and the other which activates $\mathrm{G}_{\mathrm{i}}$ but, in contrast to the present approach and to experimental observations, they do not consider any interconversion through reversible phosphorylation between these two receptor states.
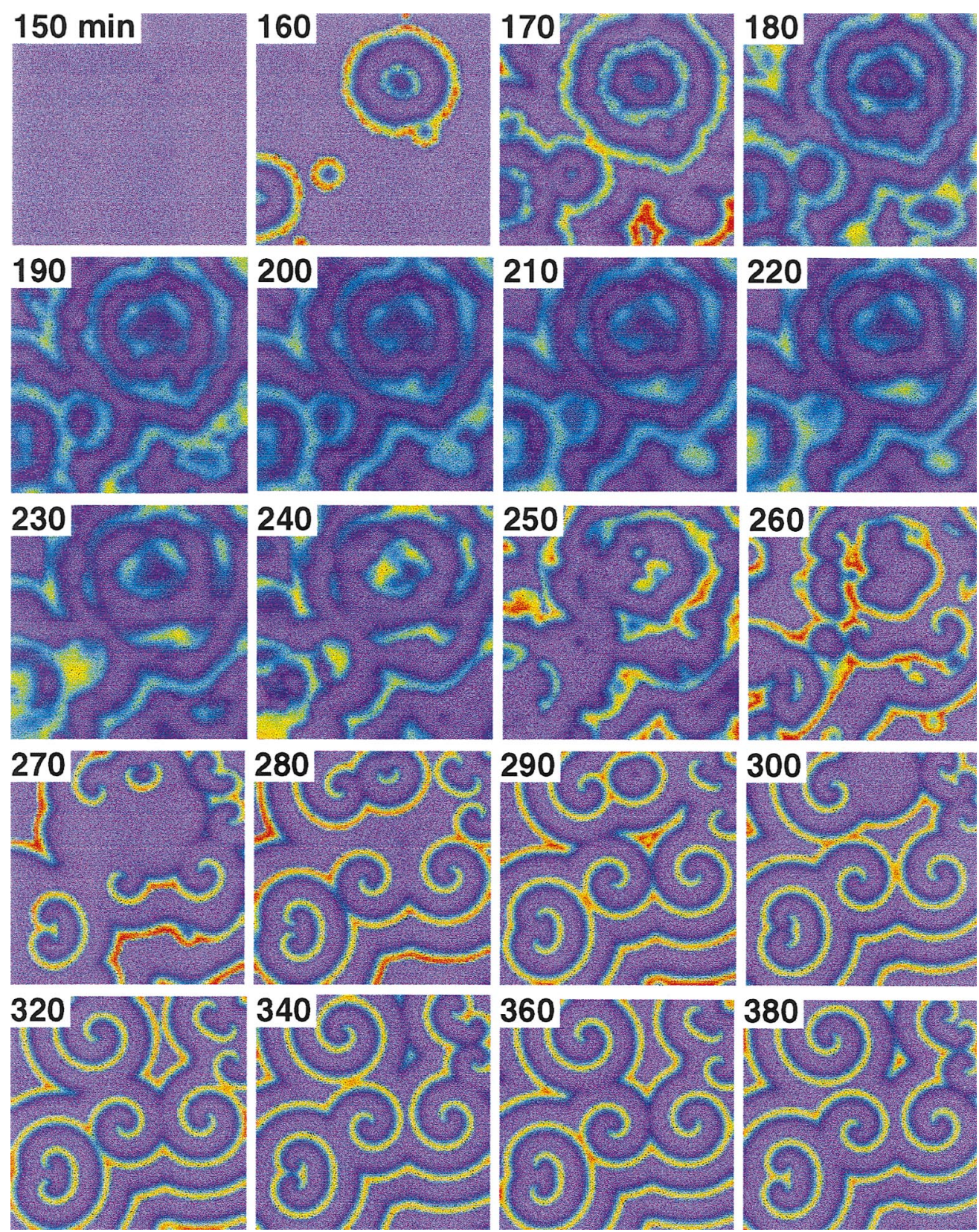

Fig. 7. Detailed sequence of events leading to the formation of spiral waves of cAMP. The situation considered in panel $b$ of Fig. 6 is examined here in further detail. Shown is the spatial distribution of extracellular cAMP at $10 \mathrm{~min}$ intervals, from $150 \mathrm{~min}$ to $380 \mathrm{~min}$ after the beginning of the increase in $\sigma$ and $k_{\mathrm{e}}$ on the developmental path. 
The actual control network for adenylate cyclase in D. discoideum is in fact more complex than the picture given in Fig. 2, and is far from being totally elucidated at the present time [24]. It appears that additional proteins play a role in signal transduction, both in the activation and inhibition pathways controlling cAMP signal generation in Dictyostelium cells [23]. For example, the protein cytosolic regulator of adenylate cyclase (CRAC) appears to modulate the activity of the $\mathrm{G}_{\mathrm{s}}$ protein [35] and is essential for the activation of adenylate cyclase. The results of the first part of the present study suggest, however, that the basic, three-variable form of the model incorporating reversible receptor desensitization and the positive feedback loop suffices to capture the essential aspects of cAMP relay and oscillations, regardless of the precise molecular details of adenylate cyclase activation and inhibition by the $G$ proteins. Incorporation of the latter into the model is only needed to account for the results of perfusion experiments in which the cells are subjected to controlled increments in extracellular cAMP. Given that these experiments do not correspond to the physiological conditions in which oscillations occur, it is reasonable to resort to the simpler, three-variable model in studies pertaining to the excitable and oscillatory properties of the cAMP signaling system.

The second part of our study deals with the modeling of cAMP waves which mediate $D$. discoideum aggregation after starvation. Not included in our description at this stage is the chemotactic movement of the cells. The goal of our analysis was to clarify the mechanism responsible for the formation of spiral waves of cAMP. In a previous paper [17] we focused on the possible role of cell desynchronization, and showed that the spatial heterogeneity in the evolution on the developmental path of biochemical parameters controlling the dynamic properties of the signaling system is an important factor in introducing defects which lead to the breaking of concentric waves and to their subsequent transformation into spirals. Here we extended these results by showing that the type of wave formed is markedly influenced by the degree of cellular desynchronization on the developmental path. As shown in Fig. 6, limited desynchronization leads to concentric waves, while stronger desynchronization yields a profusion of small spirals. Larger spirals are obtained for intermediate values of the degree of desynchronization $\Delta$, (see Eq. (5)). Thus, with respect to the formation of large, stable spirals, the results point to the existence of an optimal range of cellular desynchronization. The origin of such a desynchronization lies in the fact that the amoebae are caught at different phases of the cell cycle at the beginning of starvation [36]. The slowest cells lag by about $1 \mathrm{~h}$ with respect to the fastest cells in acquiring the capability of relaying cAMP signals [37] or of oscillating autonomously $[38,39]$; such a time difference holds with the values of $\Delta$, of the order of $25 \mathrm{~min}$, producing large spiral waves of cAMP [17].

We have also presented a sequence of spatiotemporal frames which provides a detailed history of the formation of cAMP spirals. The data in Fig. 7 indicate that concentric waves form at first, as some cells enter the oscillatory domain in parameter space while other cells become excitable, as a result of their progression on the developmental path. Then defects arise, which eventually break the concentric patterns, as cells begin to leave the oscillatory domain. Spirals develop as the fragmented waves curl up at their extremities. The duration of the phase of breaking the concentric waves depends both on the degree of desynchronization and on the rate at which cells progress on the developmental path.

The results on the effect of parameter $\Delta$ suggest that better synchronization of the cells after starvation should favor the formation of concentric over spiral waves [17]. However, the present results have been obtained by assuming that all cells evolve at the same rate on the same developmental path and differ only by their initial position on this path. Besides this source of desynchronization, captured by parameter $\Delta$ and by the distribution of initial times given by Eq. (5), additional factors contributing to cellular heterogeneity might be considered. Thus, the initial and final values of the enzyme concentrations (measured here by parameters $\sigma$ and $k_{\mathrm{e}}$ ) as well as the rate of evolution on the developmental path might also differ from cell to cell. Distributions of these parameters around mean values could be considered in an extension of the present work, to determine the possible influence of these sources of heterogeneity on the formation of spiral waves, even in conditions where cells are apparently synchronized and the value of parameter $\Delta$ is reduced.

The developmentally based scenario for the onset 
of spiral waves does not rule out the possible contribution of other important factors. Any process that helps to build up defects associated with spatial heterogeneities is likely to favor the formation of spirals. As shown by theoretical and experimental studies, one such factor could be the phosphodiesterase inhibitor released by the amoebae into the extracellular medium $[31,40]$, since this factor indirectly controls the level of cAMP. The effect of this inhibitor is implicitly included in the developmental path shown in Fig. 5 , since it enters the net activity of phosphodiesterase. Of particular significance is the chemotaxis of the cells, which has been considered in other theoretical studies of wavelike aggregation [16,30]. Chemotactic cell movement can lead to heterogeneities in cell density that may contribute to the formation of defects in propagating waves of cAMP. It will be instructive to include both the chemotactic movement of the amoebae and their temporal evolution on the developmental path, in a unified description of wavelike aggregation of Dictyostelium cells in response to pulsatile signals of cAMP.

\section{Acknowledgements}

This work was supported by the programme 'Actions de Recherche Concertée' (ARC 94-99/ 180) launched by the Division of Scientific Research, Ministry of Science and Education, French Community of Belgium.

\section{References}

[1] J.T. Bonner, The Cellular Slime Molds, 2nd edn., Princeton University Press, Princeton, NJ, 1967.

[2] G. Gerish, Annu. Rev. Biochem. 56 (1987) 853.

[3] P.N. Devreotes, Science 245 (1989) 1054.

[4] F. Alcantara, M. Monk, J. Gen. Microbiol. 85 (1974) 321.

[5] T.A. Durston, Dev. Biol. 37 (1974) 225.

[6] K.J. Tomchik, P.N. Devreotes, Science 212 (1981) 443.

[7] F. Siegert, C.J. Weijer, Physica D 49 (1991) 224.

[8] G. Gerish, U. Wick, Biochem. Biophys. Res. Commun. 65 (1975) 364
[9] W. Roos, V. Nanjundiah, D. Machow, G. Gerish, FEBS Lett. 53 (1975) 139.

[10] B.M. Shaffer, Nature 255 (1975) 549.

[11] A. Goldbeter, Biochemical Oscillations and Cellular Rhythms: The Molecular Bases of Periodic and Chaotic Behaviour, Cambridge University Press, Cambridge, UK, 1996.

[12] P.N. Devreotes, J. A Sherring, J. Biol. Chem. 260 (1985) 6378.

[13] P. Klein, A. Theibert, D. Fontana, P.N. Devreotes, J. Biol. Chem. 260 (1985) 1757.

[14] J.L. Martiel, A. Goldbeter, Biophys. J. 52 (1987) 807.

[15] J.J. Tyson, J.D. Murray, Development 106 (1989) 421.

[16] T. Höfer, J.A. Sherratt, P.K. Maini, Proc. R. Soc. Lond. B 259 (1995) 249.

[17] J. Lauzeral, J. Halloy, A. Goldbeter, Proc. Natl. Acad. Sci. USA 94 (1997) 9153.

[18] A.G. Gilman, Ann. Rev. Biochem 56 (1987) 615.

[19] E.J. Neer, Curr. Biol. 7 (1997) R31.

[20] B.E. Snaar-Jagalska, P.J.M. Van Haastert, Mol. Cell. Biochem. 92 (1990) 177.

[21] B.E. Snaar-Jagalska, S. Van Es, F. Kesbeke, P.J.M. Van Haastert, Eur. J. Biochem 195 (1991) 715.

[22] M.C. Chen, R.I. Insall, P.N. Devreotes, Trends Genet. 12 (1996) 52.

[23] R.A. Firtel, Curr. Opin. Genet. Dev. 6 (1996) 545.

[24] J.Y. Kim, P.J.M. Van Haastert, P.N. Devreotes, Chem. Biol. 3 (1996) 239.

[25] J. Halloy, Thèse de Doctorat en Sciences, Université Libre de Bruxelles, Brussels, 1996.

[26] Y. Tang, H.G. Othmer, Math. Biosci. 120 (1994) 25.

[27] P.N. Devreotes, T.L. Steck, J. Cell. Biol. 80 (1979) 300.

[28] P.B. Monk, H.G. Othmer, Proc. R. Soc. Lond. B 240 (1990) 555.

[29] H. Levine, I. Aronson, L. Tsimring, T.V. Truong, Proc. Natl. Acad. Sci. USA 93 (1996) 6382.

[30] J.C. Dallon, H. G Othmer, Phil. Trans. R. Soc. Lond. B 352 (1997) 1.

[31] E. Palsson, E.C. Cox, Proc. Natl. Acad. Sci. USA 93 (1996) 1151.

[32] W.F. Loomis, Dev. Biol. 70 (1979) 1.

[33] A. Goldbeter, L.A. Segel, Differentiation 17 (1980) 127.

[34] A. Goldbeter, J.L. Martiel, in: M. Markus, S. Müller, G. Nicolis (Eds.), From Chemical to Biological Organization, Springer, Berlin, 1988, p. 248.

[35] P.J. Lilly, P.N. Devreotes, J. Biol. Chem. 269 (1994) 14123.

[36] S.A. McDonald, A.J. Durston, J. Cell. Sci. 66 (1984) 195.

[37] A.R. Gingle, A. Robertson, J. Cell Sci. 20 (1976) 21.

[38] S.A. McDonald, Dev. Biol. 117 (1986) 546.

[39] R.H. Gomer, R.A. Firtel, Science 237 (1987) 758.

[40] E. Palsson, K.J. Lee, R.E. Goldstein, J. Franke, R.H. Kessin, E.C. Cox, Proc. Natl. Acad. Sci. USA 94 (1997) 13719. 